\title{
Are Birth Certificate and Hospital Discharge Linkages Performed in 52 Jurisdictions in the United States?
}

\author{
Shin Y. Kim ${ }^{1} \cdot$ Sukhjeet Ahuja $^{2} \cdot$ Caroline Stampfel $^{3} \cdot$ Dhelia Williamson $^{1}$
}

Published online: 4 July 2015

(C) Springer Science+Business Media New York (outside the USA) 2015

\begin{abstract}
Objectives The purpose of this study was to determine the number and characteristics of US State Registrars of Vital Statistics (Vital Registrars) and State Systems Development Initiative (SSDI) Coordinators that link birth certificate and hospital discharge data as well as using linkage processes.

Methods Vital Registrars and SSDI Coordinators in all 52 vital records jurisdictions (50 states, District of Columbia, and New York City) were asked to complete a 41-question survey. We examined frequency distributions among completed surveys using SAS 9.3.

Results The response rate was $100 \%(\mathrm{~N}=52)$ for Vital Registrars and $96 \%(\mathrm{~N}=50)$ for SSDI Coordinators. Nearly half of Vital Registrars $(\mathrm{n}=22)$ and SSDI Coordinators $(n=23)$ reported that their jurisdiction linked birth certificate and hospital discharge records at least once in the last 4 years. Among those who link, the majority of Vital Registrars $(77.3 \%)$ and SSDI Coordinators (82.6\%)
\end{abstract}

The findings and conclusions in this report are those of the authors and do not necessarily represent the official position of the Centers for Disease Control and Prevention.

Shin Y. Kim

skim1@cdc.gov

1 Division of Reproductive Health, National Center for Chronic Disease Prevention and Health Promotion, Centers for Disease Control and Prevention, 4770 Buford Hwy, NE MS K-23, Atlanta, GA 30341, USA

2 Health Statistics and Research, National Association for Public Health Statistics and Information Systems, Silver Spring, MD, USA

3 Association of Maternal and Child Health Programs, Washington, DC, USA link both maternal and infant hospital discharge records to the birth certificate. Of those who do not link, $43 \%$ of the Vital Registrars and $55 \%$ of SSDI Coordinators reported an interest in linking birth certificate and hospital discharge data. Reasons for not linking included lack of staff time, inability to access raw data, high cost, and unavailability of personal identifiers to link the two sources.

Conclusions Results of our analysis provide a national perspective on data linkage practices in the US. Our findings can be used to promote further data linkages, facilitate sharing of data and linkage methodologies, and identify uses of the resulting linked data.

Keywords Birth certificate $\cdot$ Hospital discharge · Linkage $\cdot$ Survey

\section{Significance}

Our survey demonstrated that linking birth certificate and hospital discharge data is feasible and that supporting linkage activities is needed to overcome barriers and challenges in building and strengthening capacity for all jurisdictions.

\section{Introduction}

Public health agencies and researchers rely heavily on birth certificate and hospital discharge data for national and local surveillance and research activities related to pregnancy complications, risk behaviors, and neonatal outcomes [1, 2]. These data sources are large, accessible, and available for nearly all in-hospital births in the United States. Birth certificates provide information about some maternal 
diagnoses, gestational age, maternal age, body mass index, gestational weight gain, and maternal smoking, while hospital discharge data provide codes for diagnoses and procedures related to labor, delivery, and pregnancy complications. Previous studies have found that combining these two databases provides a wider range of data and a more accurate source for identifying maternal diagnoses than a single source alone $[3,4]$. These linked data are also a feasible source for examining contributions of known modifiable risk factors to pregnancy complications $[5,6]$. Results from analyses using these linked administrative data can be used at state and national levels to inform maternal and child health programs, improve public health infrastructure including up-to-date data and information systems to respond to public health needs, and monitor trends in pregnancy conditions, risk behaviors, and perinatal outcomes [7-9].

Currently, the extent of administrative data linkages in jurisdictions is unknown. State Maternal and Child Health $(\mathrm{MCH})$ Services Block Grant recipients report on their $\mathrm{MCH}$ data capacity and ability to obtain timely analyses of certain data for programmatic and policy issues. For example, states report their ability to access at least $90 \%$ of in-state discharges. States do not report their ability to link hospital discharge records to birth certificate data [10]. In collaboration with the Centers for Disease Control and Prevention (CDC), the National Association for Public Health Statistics and Information System (NAPHSIS) and the Association of Maternal \& Child Health Programs (AMCHP) developed a 41-question survey. NAPHSIS is the national nonprofit organization representing the state vital records and public health statistics offices in the United States. AMCHP is a national resource, partner and advocate organization for state public health leaders and others working to improve the health of women, child, youth and families. For this survey, researchers sought to obtain responses about birth certificate and hospital discharge data linkages from two perspectives, as reported by State Registrars of Vital Statistics (also referred to as Vital Registrars) and State Systems Development Initiative (SSDI) Coordinators. The purpose of this study was twofold: to determine the number of jurisdictions that link birth certificate and hospital discharge data and to describe linkage processes used and barriers to conducting linkages.

\section{Materials and Methods}

\section{Study Population}

The study population consisted of Vital Registrars and SSDI Coordinators from the state health department in all 52 vital records jurisdictions in the United States (50 states,
District of Columbia, and New York City). Vital Registrars were chosen because they represent all US jurisdictions and are responsible for registering vital events and reporting vital statistics data to local, state, and federal partners for administrative, research, and surveillance activities. SSDI Coordinators were chosen as they also represent all US jurisdictions and because they work with state Maternal and Child Health programs to build infrastructure that results in comprehensive, community-based systems of care for all children and their families. In jurisdictions where linkages are conducted, Vital Registrars and SSDI Coordinators oversee linkage activities.

\section{Survey Design}

Using the online software Zoomerang (Survey Monkey, Palo Alto, CA), NAPHSIS administered the survey to all Vital Registrars and AMCHP administered the same survey to all SSDI Coordinators. AMCHP and NAPHSIS performed cognitive and functional pilot testing of the survey with a small subset of the target survey audience. Feedback from each round of testing was incorporated into the final survey. All survey materials and methods were approved by the Office of Management and Budget (OMB 0920-0879) and were deemed exempt by the CDC Institutional Review Board.

\section{Data Collection}

Vital Registrars and SSDI Coordinators received emails notifying them about survey sponsorship, purpose, content, and dates of administration. We asked the registrars and coordinators to complete the survey with input from staff as needed. A second email included a link to the survey along with information about software requirements, a hard copy of the survey, and detailed instructions. The survey remained open for 60 business days between September and November 2012. Reminder emails were sent to nonrespondents on days 20 and 40 and reminder phone calls were made on day 30 . No incentives were offered for completion.

Questions assessed the utility of and barriers to birth certificate and hospital discharge data linkages, including information on access to the linked birth certificate and hospital discharge data and quality of the linkage. Specifically, study subjects were asked whether any organizations in their jurisdiction, including the state health department, universities, or other organizations had linked birth records and hospital discharge records in the last 4 years. Those answering affirmatively were asked additional questions about what types of records were linked (maternal and/or infant), what methodology (deterministic-the exact matching of one or more pieces of data with identifiers or 
probabilistic_comparing records using a combination of identifiers and weights) and software were used to link, how often the linkage was updated, and whether the linkage was automated. If no linkage had been conducted, subjects were asked about barriers to and interest in linking these records with check all that apply.

\section{Data Analysis}

We coded and analyzed completed surveys using SAS 9.3 (SAS Institute Inc., Cary NC). Data analysis yielded frequency distributions and percentages for each of the survey questions. We report all results separately for the two groups.

\section{Results}

The response rate was $100 \%(\mathrm{~N}=52)$ for Vital Registrars and $96 \%(\mathrm{~N}=50)$ for SSDI Coordinators. Nearly half of Vital Registrars (42\%) and SSDI Coordinators (46\%) reported that some organization (e.g., state health department, university) linked birth certificate and hospital discharge records (maternal and/or infant) in the last 4 years. Of the 52 Vital Registrars, 26 reported that they do not link and 4 reported that they do not know whether a linkage occurs. Of the 50 SSDI Coordinators, 25 reported that they do not link and 2 reported that they do not know whether a linkage occurs.

Table 1 Data linkage practices by State Registrars of Vital Statistics and State Systems Development Initiative (SSDI) Coordinators among those who link birth certificate and hospital discharge data

\begin{tabular}{|c|c|c|}
\hline Survey questions and response options & $\begin{array}{l}\text { Vital Registrars } \\
\mathrm{N}=22 \\
\mathrm{n}(\%)\end{array}$ & $\begin{array}{l}\text { SSDI Coordinators } \\
\mathrm{N}=23 \\
\mathrm{n}(\%)\end{array}$ \\
\hline \multicolumn{3}{|c|}{ Which inpatient hospital discharge records are linked to the birth record, in the last 4 years? } \\
\hline Maternal & $2(9.1)$ & $2(8.7)$ \\
\hline Infant & $3(13.6)$ & $2(8.7)$ \\
\hline Both & $17(77.3)$ & $19(82.6)$ \\
\hline \multicolumn{3}{|c|}{ Is your linkage automated (i.e. when data are received, is there already code developed to clean and merge into full dataset)? } \\
\hline Yes & $9(40.9)$ & $11(47.8)$ \\
\hline No & $12(54.5)$ & $7(30.4)$ \\
\hline Don’t know & $1(4.5)$ & $5(21.7)$ \\
\hline \multicolumn{3}{|l|}{ How often is the linked file updated? } \\
\hline$<$ once every 2 years & $1(4.5)$ & $3(13.0)$ \\
\hline Every other year & $0(0.0)$ & $0(0.0)$ \\
\hline Annually & $12(54.5)$ & $11(47.8)$ \\
\hline More than annually & $1(4.5)$ & $3(13.0)$ \\
\hline Only once & $5(22.7)$ & $2(8.7)$ \\
\hline Other & $2(9.1)$ & $2(8.7)$ \\
\hline Don't know & $1(4.5)$ & $2(8.7)$ \\
\hline \multicolumn{3}{|c|}{ What is the methodology used for linking the records? (Check all that apply) } \\
\hline Deterministic & $13(59.1)$ & $13(56.5)$ \\
\hline Probabilistic & $12(54.5)$ & $9(39.1)$ \\
\hline Other & $2(9.1)$ & $1(4.3)$ \\
\hline \multicolumn{3}{|c|}{ Has the quality of the birth-hospital record linkage been evaluated? } \\
\hline Yes & $13(59.1)$ & $11(47.8)$ \\
\hline No & $8(36.4)$ & $5(21.7)$ \\
\hline Don’t know & $1(4.5)$ & $7(30.4)$ \\
\hline \multicolumn{3}{|c|}{ How does your state currently use your birth-hospital record linked dataset? (Check all that apply) } \\
\hline State reports & $9(40.9)$ & $12(52.2)$ \\
\hline Surveillance & $12(54.5)$ & $14(60.9)$ \\
\hline Special studies & $12(54.5)$ & $19(82.6)$ \\
\hline Policy evaluation & $4(18.2)$ & $4(17.4)$ \\
\hline Program or quality improvement & $9(40.9)$ & $13(56.5)$ \\
\hline Other & $1(4.5)$ & $1(4.3)$ \\
\hline
\end{tabular}




\section{Linkage Process, Quality Assessment Measures, and Use of Linked Data}

Of those who link their data, $77 \%$ of Vital Registrars and $83 \%$ of SSDI Coordinators reported linking both maternal and infant hospital discharge records to birth certificate records (Table 1). While, $14 \%$ of Vital Registrars and $9 \%$ of SSDI Coordinators reported linking maternal records only; about $9 \%$ of each type of respondent reported linking infant records only. Nearly half of Vital Registrars $(41 \%)$ and half SSDI Coordinators (48\%) reported having a computerized algorithm that cleans and links the data sources with no manual intervention required. Reasons for not having an automated linkage process included lack of software, a standard data format, experience, and financial resources.

Response options for the question on frequency of conducting the data linkage included (1) Less than once every 2 years; (2) Every other year; (3) Annually; (4) More frequently than annually; (5) Have only done the linkage once; (6) Other; (7) Don't know. The three highest responses among Vital Registrars were 12 reported an annual linkage and 5 reported only a one-time linkage and among SSDI Coordinators were 11 reported an annual linkage, 3 reported more than an annual linkage, and 3 reported linking less than once every 2 years (Table 1 ). Half of Vital Registrars and SSDI Coordinators reported using deterministic methodologies (exact matching of one or more pieces of data). Fifty-nine percent of Vital Registrars and $48 \%$ of SSDI Coordinators performed some type of quality evaluation of the birth certificate-hospital discharge record linkage. The majority of both groups reported using the linked data for special studies, surveillance, and quality improvement. Relatively few use the data for policy evaluation.

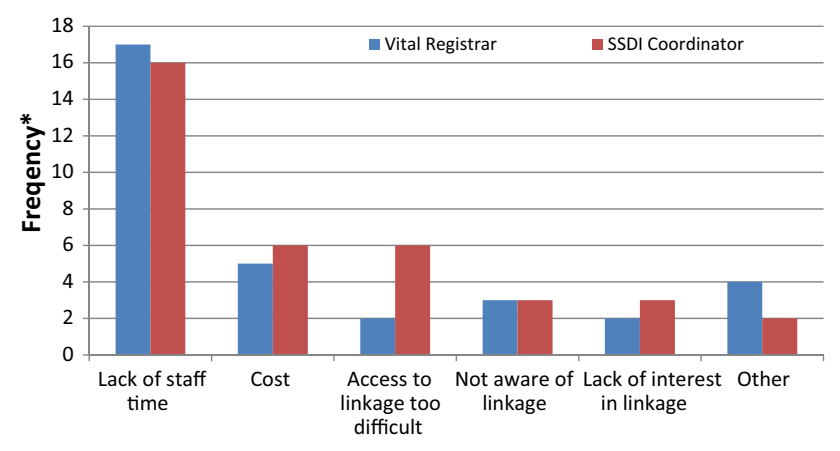

Fig. 1 Among those who link, barriers to using the linked data among State Registrars of Vital Statistics and State Systems Development Initiative (SSDI) Coordinators. Asterisk The frequency is the number of times the response was given as respondent can check more than one response

\section{Reasons Linked Data are not Used and Barriers to Conducting Linkages}

Both Vital Registrars and SSDI Coordinators reported lack of staff time followed by cost and difficult access to the data as primary reasons for not using their linked data (Fig. 1). For the response "other-specify", Vital Registrars and SSDI Coordinators wrote: "lack of access to identifiable information for linking," "inadequate executive support," and "legislative restrictions." For both Vital Registrars and SSDI Coordinators who do not conduct linkages, barriers to linking included lack of staff time, lack of access to raw data, cost, and lack of access to identifiable information for linking (Fig. 2). For the response otherspecify respondents listed legislative restrictions, privacy/confidentiality issues, and lack of availability of data items from both datasets as additional reasons for barriers.

\section{Interest in Conducting Linkages and Sharing Data Linkage Processes}

Most Vital Registrars (73\%) and SSDI Coordinators $(83 \%)$ who conduct linkages reported that they would be interested in collaborating with others to share linkage processes, lessons learned, and linkage software codes (Table 2). Reasons for those who answered "not sure" about such collaborations included time commitment, cost, confidentiality of data, and data linkage did not occur in the health department were the reasons (data not shown). Among those who do not link birth certificate-hospital discharge data, more SSDI Coordinators $(60 \%)$ than Vital Registrars (42\%) were interested in linking these data sources.

\section{Discussion}

This study extends previous work on birth certificate and hospital discharge linkages by surveying Vital Registrars and SSDI Coordinators about their birth certificate and hospital discharge data linking practices. Overall, we found that nearly half of Vital Registrars and SSDI Coordinators in the United States have conducted a linkage of these data sources during the last 4 years, information that was previously unknown. Results from this survey provide information about linkage methods, uses of the linked data, and barriers to conducting linkages. In both groups, most reported using the linked data for special studies, surveillance, and quality improvement while relatively few reported using the data for policy evaluation. Those who indicated they had protocols to automatically link the data largely were able to do so without manual intervention. Most survey respondents indicated a willingness to share 
Fig. 2 For those who do not link, barriers to conducting data linkages among State Registrars of Vital Statistics and State Systems Development Initiative (SSDI) Coordinators. Asterisk The frequency is the number of times the response was given as respondent can check more than one response

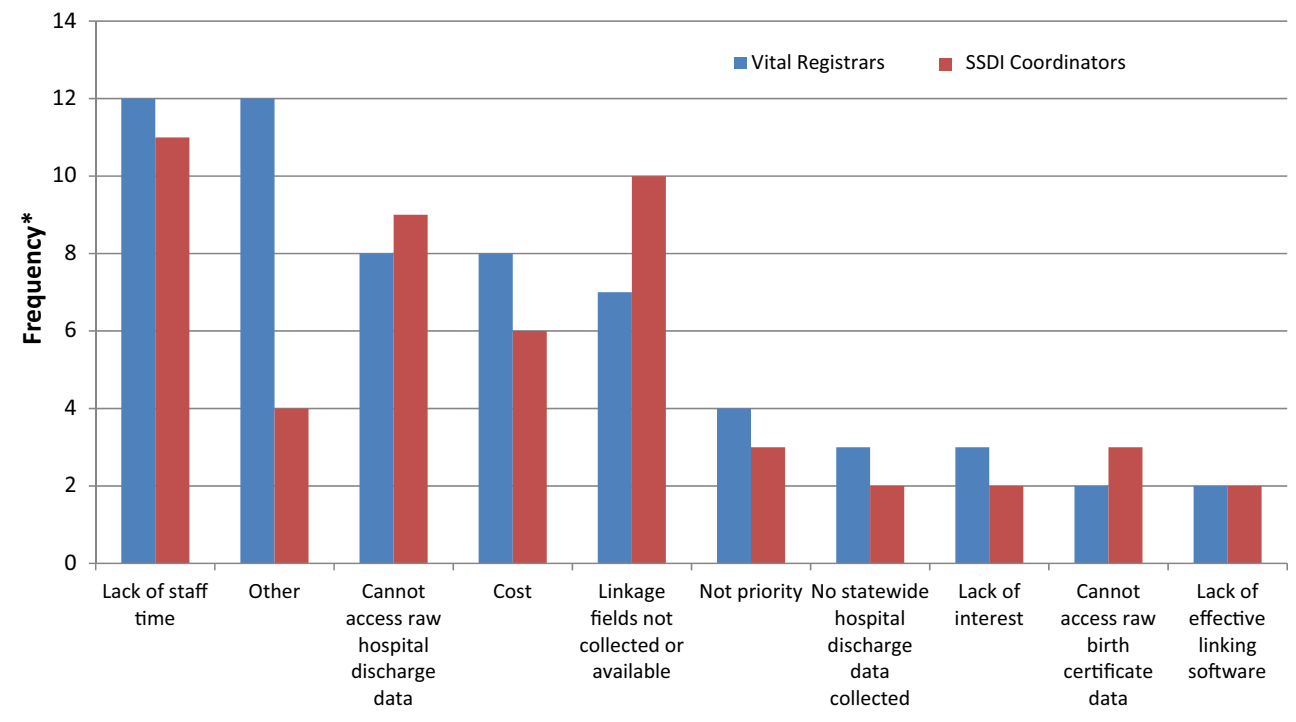

Table 2 Interest in sharing data linkage practices by State Registrars of Vital Statistics and State Systems Development Initiative (SSDI) Coordinators among those who link and do not link

\begin{tabular}{|c|c|c|}
\hline Survey questions and response options & $\begin{array}{l}\text { Vital Registrars } \\
\mathrm{n}(\%)\end{array}$ & $\begin{array}{l}\text { SSDI Coordinators } \\
\mathrm{n}(\%)\end{array}$ \\
\hline Among those who link: & $\mathrm{n}=22$ & $\mathrm{n}=23$ \\
\hline \multicolumn{3}{|c|}{$\begin{array}{l}\text { Would you be interested in collaborating with other states in sharing linkage processes, including } \\
\text { lessons learned, challenges encountered, and codes? }\end{array}$} \\
\hline Yes & $16(72.7)$ & $19(82.6)$ \\
\hline No & $0(0.0)$ & $0(0.0)$ \\
\hline Not Sure & $6(27.3)$ & $4(17.4)$ \\
\hline Among those who do not link: & $\mathrm{n}=26$ & $\mathrm{n}=25$ \\
\hline \multicolumn{3}{|c|}{ Does your organization or state want to have a linkage of birth and hospital discharge records? } \\
\hline Yes, definitely & $8(30.8)$ & $12(48.0)$ \\
\hline Yes, no rush & $3(11.5)$ & $3(12.0)$ \\
\hline Undetermined & $8(30.8)$ & $6(24.0)$ \\
\hline No, not right now & $5(19.2)$ & $4(16.0)$ \\
\hline No, never & $2(7.8)$ & $0(0.0)$ \\
\hline Among those who do not link: & $\mathrm{n}=26$ & $\mathrm{n}=25$ \\
\hline \multicolumn{3}{|c|}{ Would you be interested in collaborating with other states to learn about the linkage process? } \\
\hline Yes & $16(61.5)$ & $22(88.0)$ \\
\hline No & $7(26.9)$ & $2(8.0)$ \\
\hline Don’t know & $3(11.5)$ & $1(4.0)$ \\
\hline
\end{tabular}

these linkage algorithms and lessons learned. Sharing resources and experiences may enhance the capacity of jurisdictions that have not developed linkage protocols on their own.

While we did not present jurisdiction-level responses to preserve respondent confidentiality, differences in the percentage of aggregate data are evidence of contradictory responses to some of the same questions by Vital
Registrars and SSDI Coordinators within the same jurisdiction. Although assessing reasons for these differences was beyond the scope of this study, it is possible that Vital Registrars and SSDI Coordinators interpreted the questions differently and therefore provided different responses. As guidelines for SSDI Coordinators come from the Maternal and Child Health Bureau (MCHB), part of their responsibility includes improving surveillance systems through 
efforts such as data linkages. Therefore, linked hospital discharge and birth certificate data offer opportunities to enhance MCH surveillance. Further, it is important for states that conduct linkages to build in quality checks, validations, and standardizations into their linkage algorithms to improve reliability of linked data as well as understanding their data linkage process by examining the unmatched records [11].

The primary responsibility of the Vital Registrar is to register all vital events that occur in the jurisdiction as well as to preserve, amend, and issue certified copies of vital records in accordance with jurisdictional law. Vital Registrars also provide vital statistics data to local, state, and federal partners for administrative use, research, and surveillance activities. Therefore, they may be more likely to know if linkages occur outside of $\mathrm{MCH}$-related purposes.

\section{Conclusion}

Results from our survey shed light on the current state of administrative data linkages in the 52 US vital registration jurisdictions. Many jurisdictions are regularly linking their birth certificate and hospital discharge records and are using the linked data to inform $\mathrm{MCH}$ program and practices. To address barriers to conducting linkages and using the linked data, AMCHP and NAPHSIS plan to facilitate webinars and cross-jurisdiction trainings. In doing so, experienced states can share linkage protocols, ways to use linked data, and other lessons learned with less experienced states. Trainings could also take place at the AMCHP and NAPHSIS annual conferences and the CityMatCH Leadership and MCH Epidemiology Conference. In addition, virtual models such as a year-long analytic course, a facilitated community of practice (a collaborative framework for public health professionals), or a peer-to-peer learning network are possible approaches for assisting jurisdictions that are interested in data linkages [12].

Linked birth certificate and hospital discharge data can be used for data-driven decision-making as well as for monitoring and studying pregnancy-related complications. In a recent report, Rosenberg et al. [13] identified characteristics associated with enhanced maternal and child health epidemiology functioning in state health agencies. One of the markers of a strong data infrastructure and improved overall functioning was increasingly regular data integration (data linkage) and data sharing. Supporting linkage activities and assisting jurisdictions in addressing challenges reported in our survey are critical for building and strengthening the capacity of all jurisdictions.
Acknowledgments We thank all State Registrars of Vital Statistics and SSDI Coordinators; Patricia Potrzebowski for providing valuable feedback during the survey design and pilot phase of the project, and for her strong support of the project throughout; F. Carol Bruce for providing extensive feedback on drafts of the manuscript.

\section{References}

1. Bradford, H. M., Cardenas, V., Camacho-Carr, K., \& LydonRochelle, M. T. (2007). Accuracy of birth certificate and hospital discharge data: a certified nurse-midwife and physician comparison. Maternal and Child Health Journal, 11(6), 540-548.

2. Kim, S. Y., Sharma, A. J., Sappenfield, W., Wilson, H. G., \& Salihu, H. M. (2014). Association of maternal body mass index, excessive weight gain, and gestational diabetes mellitus with large-for-gestational-age births. Obstetrics and Gynecology, 123(4), 737-744.

3. Salemi, J. L., Tanner, J. P., Bailey, M., Mbah, A. K., \& Salihu, H. M. (2013). Creation and evaluation of a multi-layered maternal and child health database for comparative effectiveness research. J Registry Management, 40(1), 14-28.

4. Devlin, H. M., Desai, J., \& Walaszek, A. (2008). Reviewing performance of birth certificate and hospital discharge data to identify births complicated by maternal diabetes. Maternal Child Health Journal, 13(5), 660-666.

5. Lydon-Rochelle, M. T., Holt, V. L., Nelson, J. C., et al. (2005). Accuracy of reporting maternal in-hospital diagnoses and intrapartum procedures in Washington State linked birth records. Paediatric and Perinatal Epidemiology, 19(6), 460-471.

6. Kim, S. Y., Saraiva, C., Curtis, M., Wilson, H. G., Troyan, J., \& Sharma, A. J. (2013). Fraction of gestational diabetes mellitus attributable to overweight and obesity by race/ethnicity, California, 2007-2009. American Journal of Public Health, 103(10), e65-e72.

7. Jutte, D. P., Roos, L. L., \& Brownell, M. D. (2011). Administrative record linkage as a tool for public health research. Annual Review of Public Health, 32, 91-108.

8. Herrchen, B., Gould, J. B., \& Nesbitt, T. S. (1997). Vital statistics linked birth/infant death and hospital discharge record linkage for epidemiological studies. Computers and Biomedical Research, 30(4), 290-305.

9. Buehler, J. W., Prager, K., \& Hogue, C. J. (2000). The role of linked birth and infant death certificates in maternal and child health epidemiology in the United States. American Journal of Preventive Medicine, 19(1 Suppl), 3-11.

10. HRSA. Title V Information System. 2015. https://mchdata.hrsa. gov/tvisreports/MeasurementData/MeasurementDataMenu.aspx

11. Ford, J. B., Roberts, C. L., \& Taylor, L. K. (2006). Characteristics of unmatched maternal and baby records in linked birth records and hospital discharge data. Paediatric and Perinatal Epidemiology, 20(4), 329-337.

12. Communities for Public Health. [http://www.cdc.gov/phcommu nities/]. 2015.

13. Rosenberg, D., Herman-Roloff, A., Kennelly, J., \& Handler, A. (2011). Factors associated with improved MCH epidemiology functioning in state health agencies. Maternal and Child Health Journal, 15(8), 1143-1152. 